\title{
Origin of particle clustering in a simulated polymer nanocomposite and its impact on rheology
}

\author{
Francis W. Starr ${ }^{\mathrm{a})}$ and Jack F. Douglas \\ Polymers Division and Center for Theoretical and Computational Materials Science, \\ National Institute of Standards and Technology, Gaithersburg, Maryland 20899 \\ Sharon C. Glotzer \\ Departments of Chemical Engineering and Materials Science and Engineering, University of Michigan, \\ Ann Arbor, Michigan 48109
}

(Received 19 February 2003; accepted 15 April 2003)

\begin{abstract}
Many nanoparticles have short-range interactions relative to their size, and these interactions tend to be "patchy" since the interatomic spacing is comparable to the nanoparticle size. For a dispersion of such particles, it is not a priori obvious what mechanism will control the clustering of the nanoparticles, and how the clustering will be affected by tuning various control parameters. To gain insight into these questions, we perform molecular dynamics simulations of polyhedral nanoparticles in a dense bead-spring polymer melt under both quiescent and steady shear conditions. We explore the mechanism that controls nanoparticle clustering and find that the crossover from dispersed to clustered states is consistent with the predictions for equilibrium particle association or equilibrium polymerization, and that the crossover does not appear to match the expectations for first-order phase separation typical for binary mixtures in the region of the phase diagram where we can equilibrate the system. At the same time, we cannot rule out the possibility of phase separation at a lower temperature. Utilizing the existing framework for dynamic clustering transitions offers the possibility of more rationally controlling the dispersion and properties of nanocomposite materials. Finally, we examine how nanocomposite rheology depends on the state of equilibrium clustering. We find that the shear viscosity for dispersed configurations is larger than that for clustered configurations, in contrast to expectations based on macroscopic colloidal dispersions. We explain this result by the alteration of the polymer matrix properties in the vicinity of the nanoparticles. We also show that shear tends to disperse clustered nanoparticle configurations in our system, an effect particularly important for processing. (C) 2003 American Institute of Physics. [DOI: 10.1063/1.1580099]
\end{abstract}

\section{INTRODUCTION}

The rapidly expanding study of organic/inorganic nanocomposite materials offers the possibility of substantial improvements in material properties, ranging from mechanical to electrical properties, with only very small amounts of inorganic additive dispersed in an organic matrix. ${ }^{1-3}$ Dispersion of nanoparticles-such as platelike nanoclays, nanotubes, or polyhedral nanoparticles-into a polymeric matrix can have a tremendous impact on the properties of polymeric materials. Unfortunately, it often has proved difficult to form stable dispersions of nanoparticles in polymer matrices, slowing the rate of progress.

The proper characterization of these materials is further complicated by the fact that the physically relevant length scales range from macroscopic to molecular dimensions. As a result, simulations, or an analytic solution, of model nanosystems that provide insight into basic scientific questions and processes are an important tool to be exploited. The experimental complications that make quantifying particle dispersion challenging pose no barrier to molecular simula-

\footnotetext{
a) Present address: Department of Physics, Wesleyan University, Middletown, CT 06459. Electronic mail: fstarr@wesleyan.edu
}

tions, which offer direct access to the particle coordinates from which the state of dispersion can be obtained and the molecular factors that control dispersion elucidated. Simulations thus provide an opportunity to quantify how thermodynamic and processing variables or parameters, such as temperature $T$, loading $\phi$, particle-polymer interaction strength, and shear, affect the state of dispersion and the bulk nanocomposite properties. Moreover, we expect that the particle clustering strongly affects nanocomposite properties and may be an example of ubiquitous clustering phenomena-as observed in natural materials (e.g., wool, silk, cotton), biological systems (e.g., actin and fibrin), and traditional condensed phase materials (e.g., thermo-reversible gels, colloidal suspensions, equilibrium polymerization) ${ }^{4-7}$ Hence it is valuable to analyze how clustering properties of nanocomposites fit in this broad range of systems, so that knowledge from these seemingly unrelated systems can be integrated to aid the development of new nanostructured materials.

Given the range of possible nanoparticle interactions, there are a variety of possible mechanisms that might control the clustering and dispersion of nanoparticles. It is not $a$ priori clear whether nanoparticle clustering should arise from ordinary phase separation (first-order for noncritical 
concentrations) as is common in binary mixtures, or from a dynamic clustering, as in the self-organization of micelles, ${ }^{8}$ that does not involve any thermodynamic discontinuity, or from some other unexpected mechanism. Simulation can help to distinguish the mechanism for dispersion in such systems, within the limitation of the model complexity and the time and length scales accessible.

Previous simulations have elucidated the detailed changes in structure and dynamics that occur near a nanoparticle surface ${ }^{9-12}$ and possible mechanisms for reinforcement, ${ }^{13}$ and theoretical approaches used to model colloidal systems have been adapted to predict morphologies of claylike nanocomposites. ${ }^{14}$ Building on these works, we focus our attention on characterizing particle dispersion and bulk composite properties using molecular dynamics simulations of model nanoparticles embedded in a dense melt of unentangled polymers. To capture the qualitative effects of atomic structure, the model nanoparticle consists of a collection of force sites bonded together to form an icosahedral particle, whose diameter is roughly equal to the radius of gyration of the surrounding chains. This model nanoparticle resembles a $\mathrm{C}_{60}$ "Buckyball," but with fewer facets. We probe the effect of $\phi, T$, and interaction strength on the state of particle dispersion. In doing so, we use specific heat, combined with the potential energy, as a reliable metric to indicate the state of dispersion; similar experimental measurements may also be sensitive to particle clustering. We carefully characterize the particle clustering to determine the mechanism of dispersion. Our results suggest that the crossover between clustered and dispersed states is not analogous to the phase separation of a binary mixture, which we rationalize by comparing with $\mathrm{C}_{60}$ and colloidal systems. ${ }^{15,16}$ The nanoparticles appear to undergo a reversible and continuous crossover from dispersed to clustered states. The features of the clustering, such as the specific heat behavior, are consistent with the theoretical predictions made for the clustering transition that occurs in equilibrium polymerization, ${ }^{17}$ providing a link between nanocomposite dispersion and simpler thermoreversible clustering transitions. By shearing equilibrium systems where the nanoparticles are initially clustered or dispersed, we show how shear effects the state of dispersion, and hence the positions of the clustering boundaries. We also find that significant differences in viscosity of the nanocomposite only appear at relatively high loading, which we speculate may be related to geometrical considerations of the nanoparticle filler.

We organize the paper as follows: In Sec. II we describe the details of the simulation model and method. We focus on quantifying the factors that affect dispersion and the mechanism controlling clustering in Sec. III. In Sec. IV we consider the effects of shear on the nanocomposite and the resulting rheological properties.

\section{SIMULATIONS}

We perform molecular dynamics simulations of nanoparticles in a dense polymer melt. We use a well-studied beadspring model ${ }^{18-20}$ which models polymers as chains of monomers. All monomer pairs interact via a Lennard-Jones $(\mathrm{LJ})$ potential $U_{\mathrm{LJ}}$ that has been truncated at $2.5 \sigma$, where $\sigma$ is the length scale parameter of the LJ potential, and shifted so that the potential and force both smoothly vanish at the cutoff. Bonded monomers along a chain are connected via a finitely extensible, nonlinear elastic (FENE) spring potential,

$$
U_{\mathrm{FENE}}(r)=-k\left(R_{0}^{2} / 2\right) \ln \left(1-\left(r / R_{0}\right)^{2}\right),
$$

where $r$ is the distance between neighboring monomers, and $k=30$ and $R_{0}=1.5$ are adjustable parameters that have been chosen as in Ref. 9. Since we do not aim to study a specific polymer, we use reduced units in which $m=\sigma=\varepsilon=1$ ( $\varepsilon$ is the LJ energy parameter and $m$ is the monomer mass). Similarly, the length is in units of $\sigma$, the time in units of $\sigma \sqrt{m / \varepsilon}$, and the temperature in units of $\varepsilon / k_{\mathrm{B}}$ ( $k_{\mathrm{B}}$ is Boltzmann's constant). In a number of the figures, we use error bars to represent the statistical uncertainty in the calculations; these uncertainties are obtained from fluctuations of the time averages.

In prior work, ${ }^{9}$ a single nanoparticle was modeled as a collection of $356 \mathrm{LJ}$ particles bonded together in an icosahedral geometry. This geometry was chosen in order to capture general features typical of both traditional filler particles, 1,21 such as a primary carbon black particle, and nanoparticles of polyhedral geometry. ${ }^{2,22-25}$ To study many such nanoparticles in a dense melt at a reasonable loading would involve $O\left(10^{5}\right)$ particles, and hence would require extremely large amounts of CPU time, particularly given the slow dynamics involved in clustering or dispersing of the nanoparticles. While this is feasible for a few select systems, it would severely limit the scope of parameters we can explore.

As a compromise, we maintain icosahedral geometry, but use only $13 \mathrm{LJ}$ particles - one at each of the 12 vertices of the icosahedron, and one at the center. The resulting icosahedron has a diameter approximately equal to the radius of gyration of the chains comprising the melt. Assuming a reasonable value for $\sigma \approx 1 \mathrm{~nm}$, this corresponds to a nanoparticle with an approximate diameter of $3 \mathrm{~nm}$. The interaction strength between nanoparticle sites is $\varepsilon_{\mathrm{pp}}=2$, and the interaction strength between the particle sites and monomers of the chains $\varepsilon_{\mathrm{mp}}$ is varied to explore the role of polymernanoparticle interactions on clustering. Similar results are possible by holding polymer-nanoparticle interactions fixed while varying the interactions between nanoparticles. The dominant factor controlling clustering is the ratio $\varepsilon_{\mathrm{mp}} / \varepsilon_{\mathrm{pp}}$; however, the details will depend on which parameter is varied.

To maintain the icosahedral shape, the particle at each vertex is bonded to the 5 neighboring vertices and the central particle via a harmonic spring potential,

$$
U_{\text {harm }}=\kappa r_{0}^{2} / 2\left(r / r_{0}-1\right)^{2} \text {. }
$$

Between vertices, we take the bond strength $\kappa=60$ and the preferred bond length $r_{0}$ we take to be the minimum of the force-shifted Lennard-Jones potential, approximately $2^{1 / 6}$. We choose the harmonic potential because it allows to easily choose the preferred bond distance to equal the preferred Lennard-Jones distance, further ensuring the stability of the icosahedral shape. For bonds between the vertices and the central particle, we use Eq. (2) with the same value of $\kappa$, but to maintain the geometry of the icosahedron we use a 


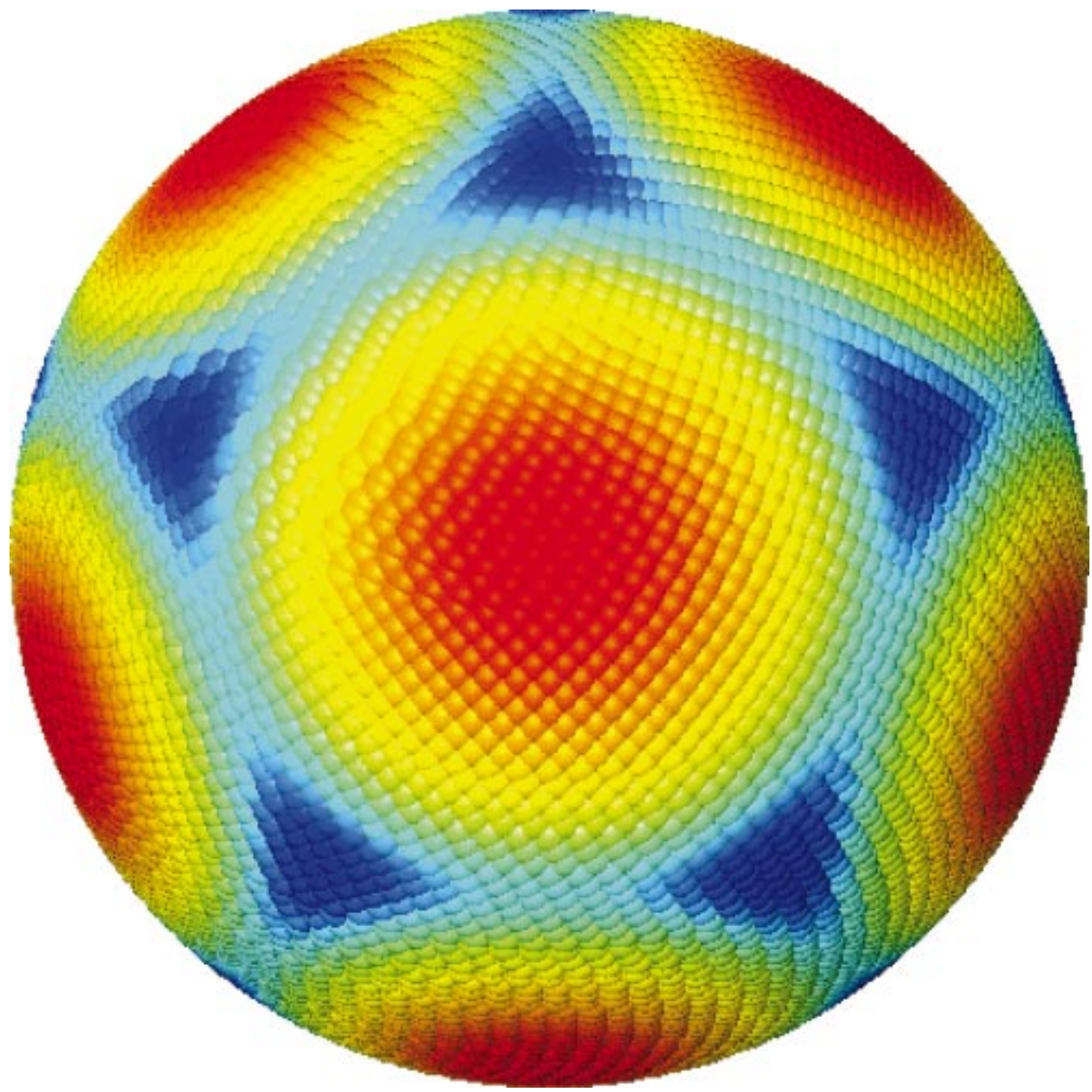

FIG. 1. (Color) Color map of the value of the nanoparticle potential energy for a test monomer a distance 1.7 from the particle center. Low energy is blue, and high energy is red. The red regions coincide with the location of the particles at the vertices, while the "sticky" blue regions correspond to centers of faces of the nanoparticle. slightly smaller preferred bond length, equal to the radius of the sphere circumscribed around the icosahedron $r_{0}^{\prime}$ $=1 / 4(10+2 \sqrt{5})^{1 / 2} r_{0}$. The resulting particles have some flexibility, but are largely rigid.

While these particles are highly symmetric, the presence of discrete force sites at the vertices results in "patchiness" of the nanoparticle surface, as illustrated in Fig. 1 by a color map of the potential between the nanoparticle and a test monomer a distance of 1.7 from the center of the nanoparticle. We expect such patchiness for real nanoparticles since the interatomic spacing of the constituent atoms of the nanoparticle is significant compared with its size. For the icosahedral nanoparticles we model, this leads to a relative energy minimum when a monomer of a chain or other nanoparticle approaches the center of a face of the icosahedron. Liquids with such directional interactions often exhibit rich clustering and association properties, as we will report here. Given the polyhedral structure and van der Waals interactions between nanoparticles, we expect their properties to be similar to those of $\mathrm{C}_{60}$ Buckyballs.

We simulate systems with 400 chains of $M=10$ monomers each (a total of $N=4000$ monomers) containing 15, 32, 64 , or 125 nanoparticles, corresponding to a loading 0.046 $<\phi<0.289$; we define $\phi \equiv N_{p} /\left(N+N_{p}\right)$, where $N_{p}$ is the total number of force sites comprising the nanoparticles. The systems are prepared by first equilibrating the pure melt, and subsequently growing cavities with cubic symmetry through- out the melt into which the nanoparticles are inserted. The system is then allowed to relax to temperature $T=2.0$ and pressure $P=1.0$, where the desired $P$ and $T$ are obtained by using the simple Berendsen scaling method. ${ }^{27}$ We choose a relatively high $T$ because the time scale on which the nanoparticles cluster or disperse is extremely long compared to the diffusion time of the monomers, which increases rapidly with decreasing $T$. From this equilibration phase, we obtain the average density $\langle\rho\rangle$ at a $T=1$ and $P=1$.

We run subsequent production simulations at a fixed $\rho$, corresponding to $\langle\rho\rangle$ obtained in the constant pressure equilibration step. Hence, the results we report are NVT simulations with $\langle P\rangle_{\mathrm{NVT}}=1.0$, and $T$ is controlled using the NoseHoover method (details below). These equilibrated configurations are also used as starting configurations for different $T$ at the same $\phi$ and $\varepsilon_{\mathrm{mp}}$; we must repeat both the thermal and pressure relaxation to achieve the new equilibrated state. Equilibration typically requires a minimum of $10^{7}$ time steps even at high $T$, because the dynamics of particle clustering or dispersion are extremely slow. Production runs to gather thermodynamic or dynamic properties are typically just as long, and sometimes significantly longer, since large fluctuations near the crossover from dispersed to clustered states require very large data samples to obtain reliable time averages.

The equations of motion are integrated via the reversible reference system propagator algorithm (rRESPA), a multiple 
time step algorithm to improve simulation speed; ${ }^{26}$ we use a basic time step of 0.002 , and use a velocity Verlet version of rRESPA with the forces separated into bonded (fast) and nonbonded (slow) components. For each update of the nonbonded forces, there are three updates of the bond forces. The temperature in production runs is controlled using the Nose-Hoover method. ${ }^{27}$ We select the "mass" of the thermostat $Q=6 N T / \omega^{2}$ where $\omega=234.09$ is the intrinsic frequency obtained from a theoretical calculation for a facecentered cubic LJ system, ${ }^{28}$ a natural frequency for the heat bath. To study the effects of shear, we use a nonequilibrium molecular dynamics (NEMD) algorithm that solves the SLLOD equations of motion for a system under planar Couette flow. ${ }^{29}$ We limit ourselves to relatively low shear rates $\dot{\gamma} \lesssim 0.5$ since larger shear rates require a very small time step to avoid breaking polymer bonds, which causes the simulation to be unstable. Such effects can also be avoided by using a stronger coupling constant in the FENE bond potential, but this again requires the use of very small time steps. ${ }^{30}$ The NEMD runs vary in length from $2 \times 10^{6}$ at high shear rates where viscosity fluctuations are small, to $10^{7}$ time steps at low shear rates where long runs are needed to reliably estimate viscosity.

\section{NANOPARTICLE DISPERSION}

\section{A. Quantifying dispersion}

The state of dispersion is affected by a number of factors, including particle loading, interparticle interactions, and temperature. Before we can explore the relative importance of these control parameters, we first must have a reliable metric to determine the state of dispersion. Indeed, a generic, reliable, and simple measurement of particle dispersion is a significant experimental barrier to the development of new nanoparticle materials. In the context of simulations, we cannot solve this problem, but we can offer some insights into quantities that are significantly affected by the state of dispersion. Below, we show that dispersion can be characterized both by structural criteria, such as scattering functions, as well as thermodynamic measures, such as specific heat, potentially accessible to experiments.

The most obvious way to determine the state of particle dispersion is visual; since we have direct access to the particle coordinates, we can easily visualize the nanoparticles without the distraction of the surrounding polymer matrix. While this is the "ultimate" test we use, our aim is to identify a simple and reliable measure of particle dispersion. Given that the morphology of the system is significantly different for clustered and dispersed states, one possibility is to use an explicit measure of structure, such as the pair correlation function $g(r)$ or the structure factor $S(q)$ (which are related via a Fourier transform). While we will consider the behavior of such measures, our goal is to use a single numerical value representative of the degree of clustering.

Computer simulations of simple liquids have shown that the potential energy $U$ is strongly sensitive to changes in local packing. ${ }^{31}$ Simulation offers the advantage that the potential energy, including the components due to each species in the system, is readily available. As the degree of disper-

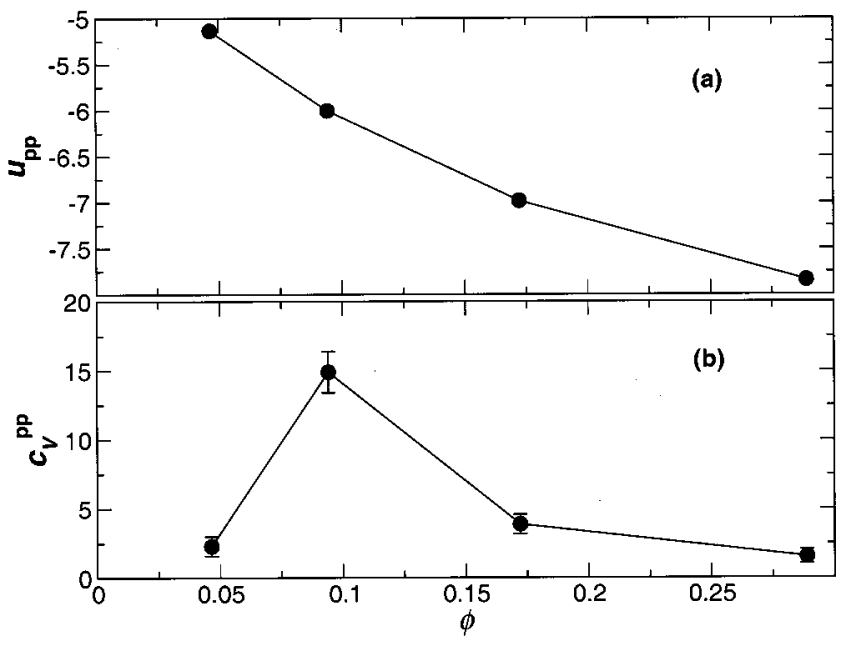

FIG. 2. (a) A nanoparticle-nanoparticle component of the potential energy $u_{\mathrm{pp}}$ at fixed $T=2.0$ and $\varepsilon_{\mathrm{mp}}=1.3$. (b) The specific heat due to nanoparticle interactions $c_{V}^{\mathrm{pp}}$ shows a clear maximum that can be used as an approximate indicator of the bounds of clustered and dispersed states.

sion changes, the most significant changes in potential energy are expected in the particle-particle $U_{\mathrm{pp}}$ and monomerparticle $U_{\mathrm{mp}}$ components of $U$, which reflect the difference in the number of particle-particle or particle-monomer contacts in the system. We will focus on $U_{\mathrm{pp}}$, sensitive to the number of particle-particle contacts. To remove trivial changes in $U_{\mathrm{pp}}$ due to changing $\phi$ we focus on the potential energy per force site $u_{\mathrm{pp}} \equiv U_{\mathrm{pp}} / N_{p}$.

To illustrate the effectiveness of the measure, we must compare the behavior of $u_{\mathrm{pp}}$ in clustered and dispersed states. To vary the state of dispersion, we vary the loading $\phi$ of nanoparticles in the system, since the limits of $\phi=0$ and $\phi=1$ must correspond to disperse and clustered states, respectively. Figure 2(a) shows $u_{\mathrm{pp}}$ at four loadings at fixed $T=2.0$ and $\varepsilon_{\mathrm{mp}}=1.3$; increasing $\phi$ leads to a decrease in $u_{\mathrm{pp}}$ as relatively more contacts are made, consistent with our expectations. To confirm that the results of Fig. 2(a) imply the existence of clustered and dispersed states, we show a snapshot of the system at loading fractions $\phi=0.046$ and 0.172 in Fig. 3; the nanoparticles are well dispersed at low $\phi$, while they are clustered at large $\phi$. It is interesting to note that in the clustered configuration $(\phi=0.172)$, a few of the nanoparticles are dispersed. This visually demonstrates the dynamic nature of the clustering that we will discuss in detail in the next section.

While these results are consistent with our expectations regarding particle clustering, it is unclear from Fig. 2(a) how to determine an approximate "boundary" between the clustered and dispersed states. To address these considerations, we consider the potential energy fluctuations $\delta u_{\mathrm{pp}} \equiv u_{\mathrm{pp}}$ $-\left\langle u_{\mathrm{pp}}\right\rangle$, which are thermodynamically quantified by the specific heat per particle of the nanoparticles,

$$
c_{V}^{\mathrm{pp}}=\frac{N_{p}\left\langle\delta u_{\mathrm{pp}}^{2}\right\rangle}{T^{2}} .
$$

At the largest and smallest loading, there are little fluctuations in potential energy, since the "phases" are highly stable. However, for parameter values between the limiting 


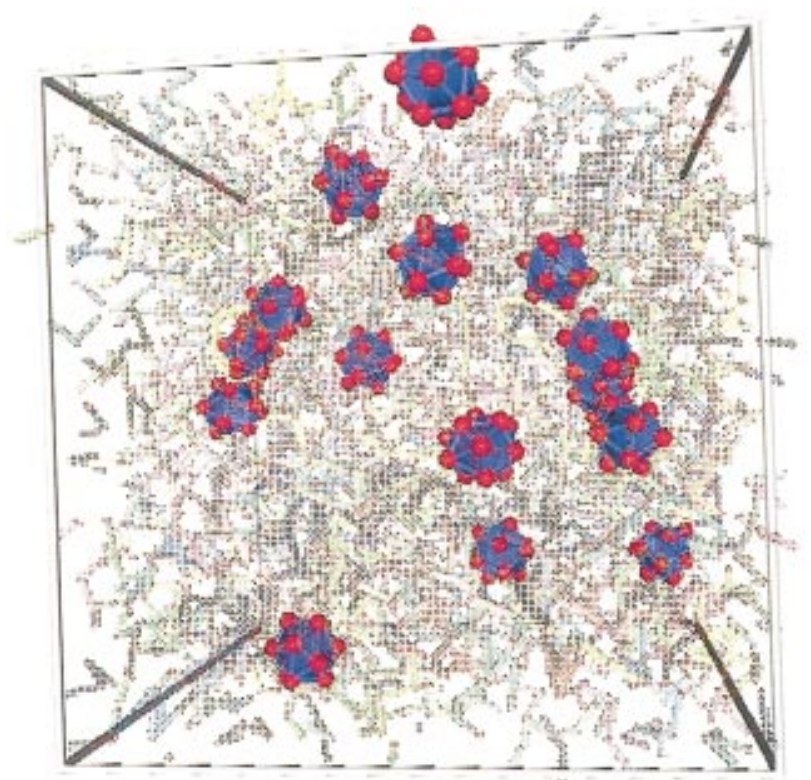

(a)

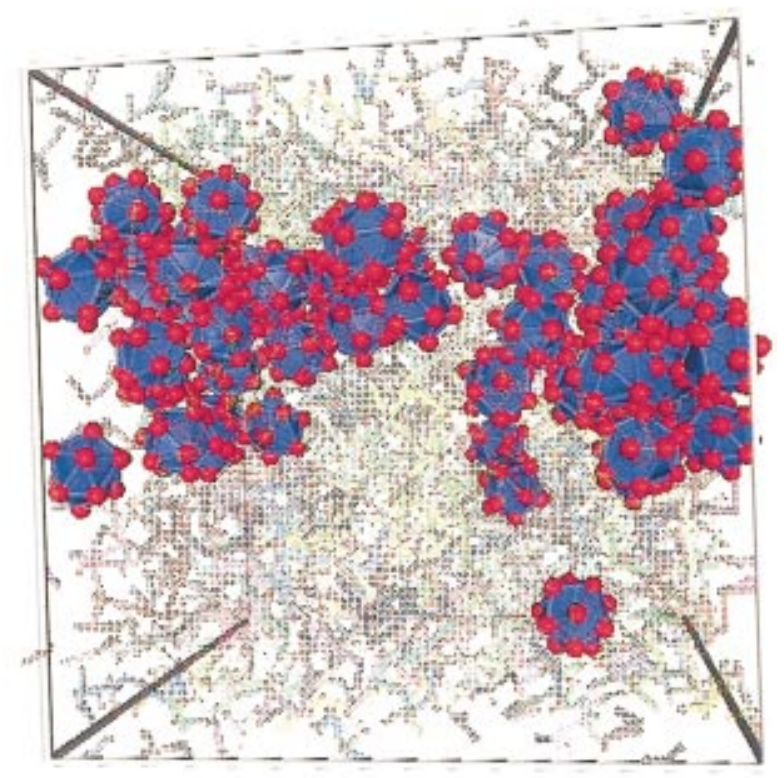

(b)

FIG. 3. (a) (Color) A simulation snapshot for $\phi=0.046$ to identify that the nanoparticles in this system are well-dispersed. (b) A snapshot at $\phi$ $=0.172$ showing the clustering of the nanoparticles. The chains are transparent to avoid obscuring the nanoparticles. The small red spheres represent the locations of the Lennard-Jones force sites.

states, particles can aggregate into small, short-lived clusters, resulting in large fluctuations in potential energy, and hence large $c_{V}^{\mathrm{pp}}$. To illustrate this, we plot $c_{V}^{\mathrm{pp}}$ for the same loadings as $u_{\mathrm{pp}}$ in Fig. 2(b), and find a pronounced maximum at intermediate loading. We emphasize that these results are not adequate to establish the nature of the transition; in the next section we carefully address this question. While the exact location of the maximum is not clear from this plot, the results illustrate that we can identify both the state of particle clustering, as well as the approximate boundary between the
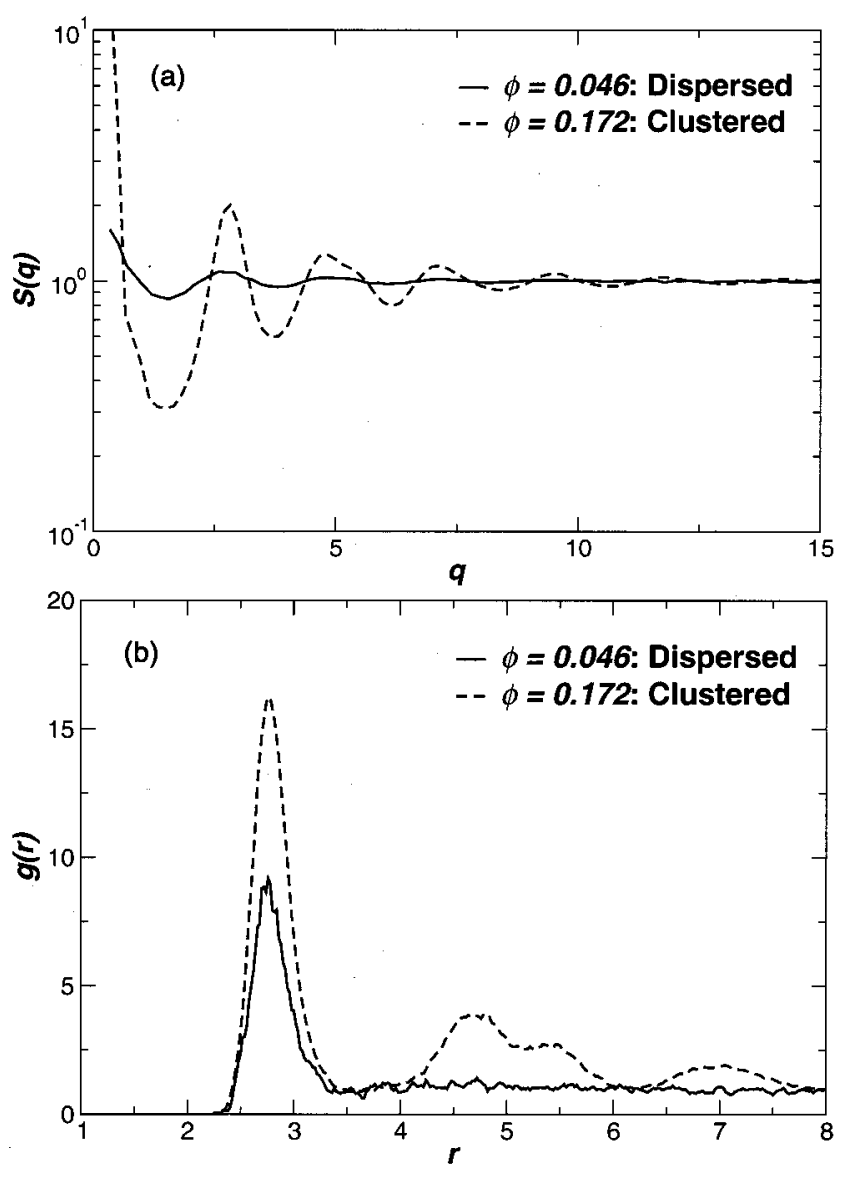

FIG. 4. (a) The structure factor $S(q)$ between the nanoparticle centers of mass. Clustered configurations show liquidlike behavior, with the addition of a strong increase at small $q$ due to longer range order. (b) The pair correlation function $g(r)$ of the nanoparticle center or mass.

states by examining $u_{\mathrm{pp}}$ and $c_{V}^{\mathrm{pp}}$. These measures will be our primary tools for determining the degree of particle clustering. The drawback is that it cannot be easily probed experimentally, due to the relative contributions to specific heat from the matrix versus the nanoparticle.

Given the dramatic difference in the visual appearance of clustered and dispersed states, traditional experimental measures, like the static structure factor,

$$
S(q)=\frac{1}{N} \sum_{j, k}^{N} e^{i \mathbf{q} \cdot\left(\mathbf{r}_{j}-\mathbf{r}_{k}\right)},
$$

must also reflect the difference. Indeed, Fig. 4(a) shows that $S(q)$ of the centers of mass of the nanoparticles in the clustered configurations at small $q$ is much larger than for disperse configurations. This can be understood from the fact that, for dispersed configurations, the nanoparticle density is more spatially homogeneous than for clustered configurations. At larger $q$, we see oscillations in $S(q)$ for the clustered system, similar to a liquid; for the dispersed system, $S(q)$ rapidly approaches 1 , similar to the behavior of a gas. While $S(q)$ is an unambiguous tool to distinguish structure at the extremes of dispersion and clustering, it requires a range of $q$ and, from an experimental standpoint, is only practical at a limited number of facilities. Additionally, there 


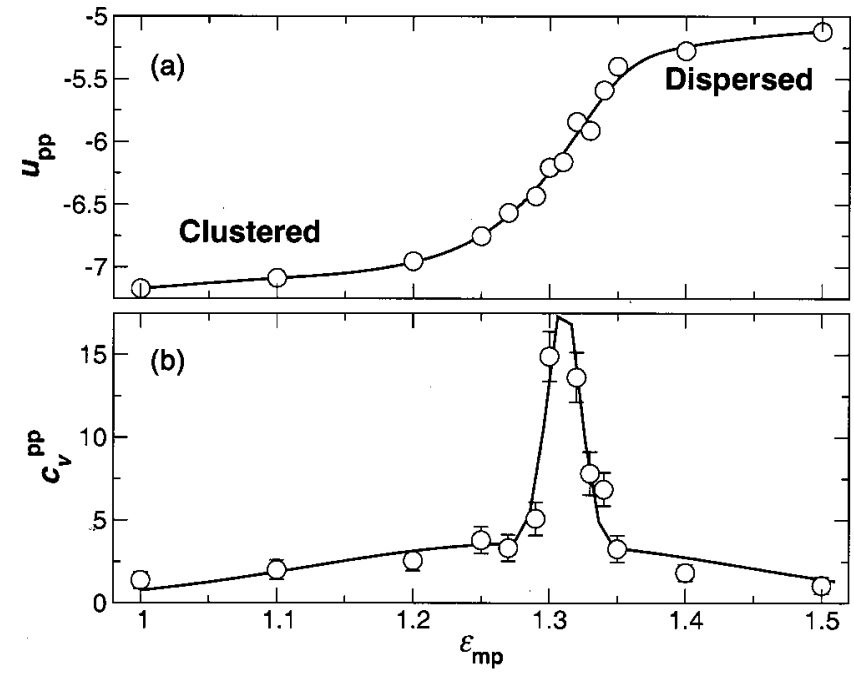

FIG. 5. (a) The nanoparticle component of the potential energy, clearly showing the crossover between clustered (low $u_{\mathrm{pp}}$ ) and dispersed (high $u_{\mathrm{pp}}$ ) states. Since the $T$ is held constant, changes in $u_{\mathrm{pp}}$ are dominated by the clustering of the nanoparticles. (b) The specific heat $c_{V}^{\mathrm{pp}}$ maximum provides a more reliable estimate of the crossover point between clustered and dispersed states.

is no clear way to delineate the approximate boundary between clustered and dispersed states using $S(q)$, as there is with $c_{V}^{\mathrm{pp}}$. Hence $c_{V}^{\mathrm{pp}}$ appears to be both simpler and more effective than $S(q)$ at identifying the state of particle clustering. We also show the pair correlation function $g(r)$, defined by the Fourier transform of $S(q)-1$ in Fig. 4(b), which shows the real space correlation of the nanoparticle centers.

\section{B. Effect of interactions}

Particle clustering can be induced in a variety of ways, such as increasing $\phi$, as in the previous section, by varying interaction, or changing $T$. The interaction between nanoparticles and the surrounding polymer matrix is likely the most important factor controlling the state of dispersion. Moreover, varying polymer-nanoparticle interactions while keeping $\phi$ fixed enables us to characterize the clustering properties of the nanocomposite without trivial clustering due to increasing $\phi$. Varying $\varepsilon_{\mathrm{mp}}$ has much the same effect of clustering as varying $T$, not surprising since $T$ is defined in terms of $\varepsilon$. However, varying $\varepsilon_{\mathrm{mp}}$ while holding $T$ fixed eliminates trivial changes in thermal energy that can obscure changes in properties due to the state of particle clustering. It is already appreciated that clustering occurs for sufficiently weak monomer-particle interactions, as well as for very strong particle-particle interactions. For the relatively simple system we study, our aim is to determine the range of interactions for which clustering or dispersion occurs, and to further characterize the crossover between these states.

To carefully characterize the nature of the crossover from clustered to dispersed states, we vary the strength of the monomer-particle interaction strength $\varepsilon_{\mathrm{mp}}$ and monitor the state of dispersion using $u_{\mathrm{pp}}$ and $c_{V}^{\mathrm{pp}}$ while holding fixed $\phi$ $=0.094$ and $T=2.0$. Figure 5(a) shows $u_{\mathrm{pp}}$ is nearly constant approaching the limits of the parameters explored, and makes a relatively abrupt crossover between two extremes

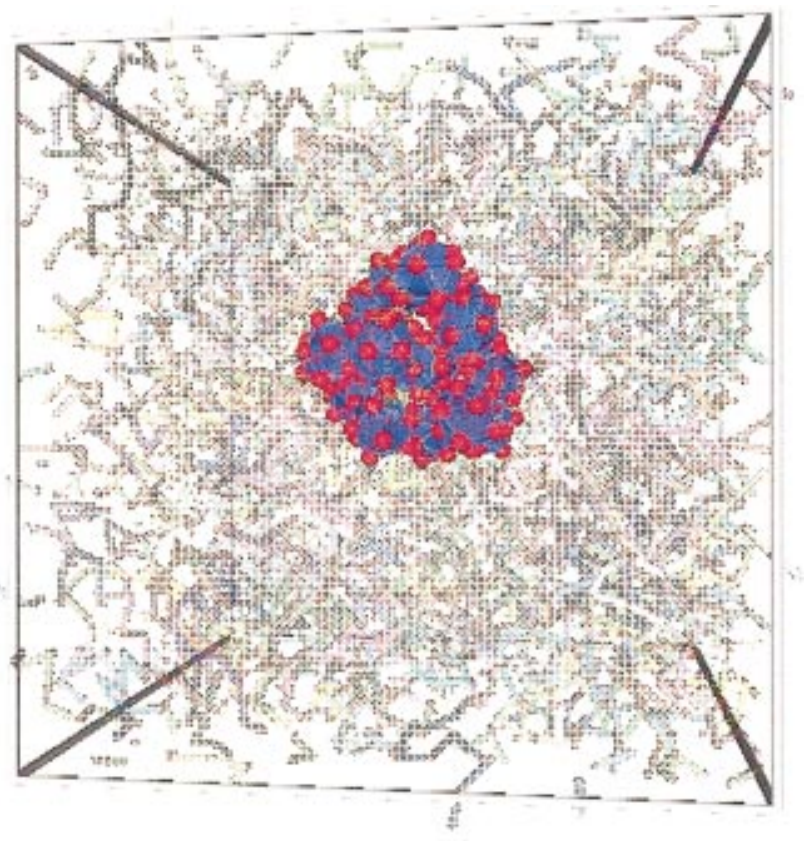

(a)

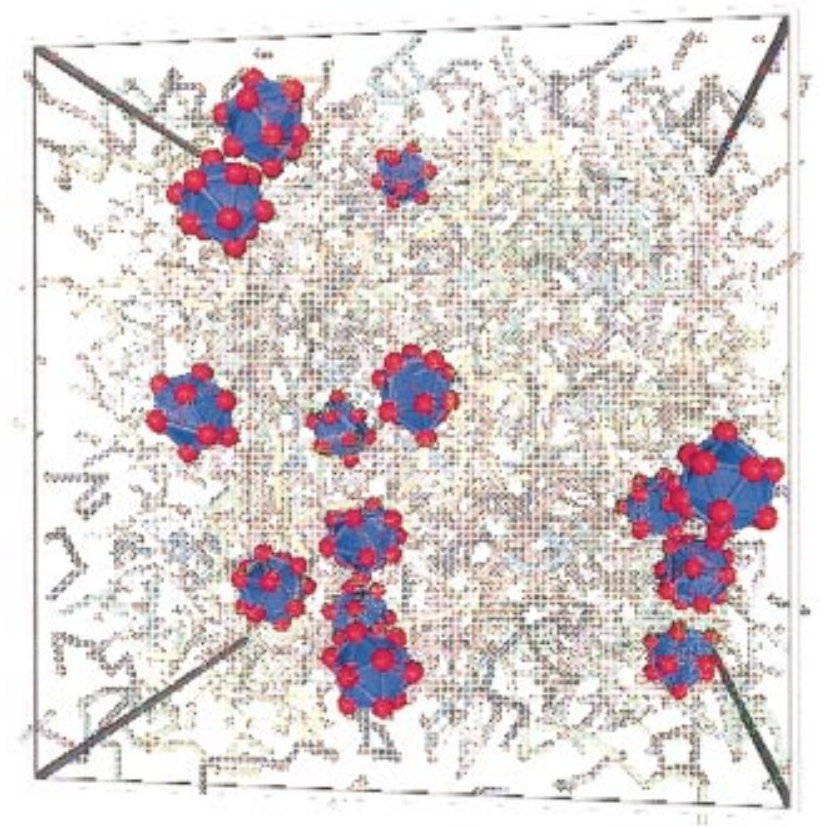

(b)

FIG. 6. (Color) Simulation snapshots of for $T=2.0$ and $\phi=0.046$. (a) shows that the nanoparticles are clustered for a relatively weak interaction $\varepsilon_{\mathrm{mp}}=1.0$. Similarly, (b) shows that dispersion occurs for $\varepsilon_{\mathrm{mp}}=1.5$. The chains are transparent to avoid obscuring the nanoparticles. The small red spheres represent the locations of the Lennard-Jones force sites.

over a narrow range $1.25<\varepsilon_{\mathrm{mp}}<1.35$. Visual inspection of the configurations (Fig. 6) at the extreme values of $\varepsilon_{\mathrm{mp}}$ confirms that the low values of $u_{\mathrm{pp}}$ correspond to clustered states, while the larger values correspond to dispersed configurations. Hence, for this $(T, \phi), \varepsilon_{\mathrm{mp}}$ needs to be only slightly stronger than $\varepsilon \equiv 1$ (the monomer-monomer interactions) for the particles to disperse. 
The range over which the crossover occurs is more clearly shown by $c_{V}^{\mathrm{pp}}$ [Fig. 5(b)]. If dispersion were to be analogous to the phase separation of a binary mixture, we would expect that $u_{\mathrm{pp}}$ and $c_{V}^{\mathrm{pp}}$ would exhibit a discontinuity, provided we do not follow a path through the critical point. However, because of the finite size of our system, we expect rounding of the transition, and hence the absence of a discontinuity here cannot rule out a first-order phase transition in the thermodynamic limit. ${ }^{32}$ However, if the transition were first order, we would expect hysteresis in the vicinity of the transition-i.e., in a narrow region near the transition our results would depend on in which direction we approach the transition. We tested this possibility, and found no evidence of hysteresis; such hysteresis can also be suppressed by a weak magnitude of the transition and finite size effects. These results suggests the transition is not first order, but our results are not conclusive; we provide more evidence supporting this possibility in the next section when we consider the specific heat of our system in relation to the pattern of behavior expected for phase separation. We also point out that our results do not, of course, preclude the existence of a first-order transition in a region of the phase diagram that we cannot explore due to the extremely slow relaxation of the system at lower $T$.

As we mentioned at the beginning of this work, it is not obvious whether clustering in our nanoparticle system should occur via a phase transition or by an equilibrium (or nonequilibrium) clustering process. We can rationalize the possible suppression of a first-order thermodynamic phase transition by focusing on the relative size of a nanoparticle (the excluded volume) and the range of interactions. In colloidal systems it has been found that an ordinary liquid-gas transition can be suppressed, depending on the ratio of the hardcore diameter $\sigma_{\mathrm{HS}}$ of the colloidal particle to the range $\delta$ of attraction induced by the depletion forces resulting from the polymers tethered to the particle surface. ${ }^{16}$ For values of $\delta / \sigma_{\mathrm{HS}} \leq 0.3$ the ordinary liquid-gas transition is pushed to very low $T$, and is typically absent from the stable phase diagram. While the nanoparticles we use consist of a collection of individual LJ particles in an icosahedral arrangement, we can estimate the approximate hard-core diameter by the distance at which $g(r)$ between the nanoparticle centers becomes nonzero; from this we obtain $\sigma_{\mathrm{HS}} \approx 2.5$. While the LJ particles at the nanoparticle surface have a range of attraction that extends to $r=2.5$ from the nanoparticle surface, the attraction is already reduced by an order of magnitude at $r$ $\approx 1.7$. Subtracting the approximate core size of a LJ particle, we obtain the range $\delta \approx 0.7$. Hence the ratio $\delta / \sigma_{\mathrm{HS}}$ $\approx 0.7 / 2.5=0.28$, and so it is possible to expect that a liquidgas-like transition would be suppressed, either completely or to very low $T$. By way of comparison, simulations of a model of $\mathrm{C}_{60}$, qualitatively similar to the nanoparticles we simulate, have shown that the phase diagram lacks a stable liquid-gas transition; the transition appears to be suppressed to the metastable regime at low $T$, where the the solid phase is most stable. ${ }^{15}$

If the crossover between clustered and dispersed states is not a simple phase transition, how can we characterize it? To start to address this, we focus on the the instantaneous

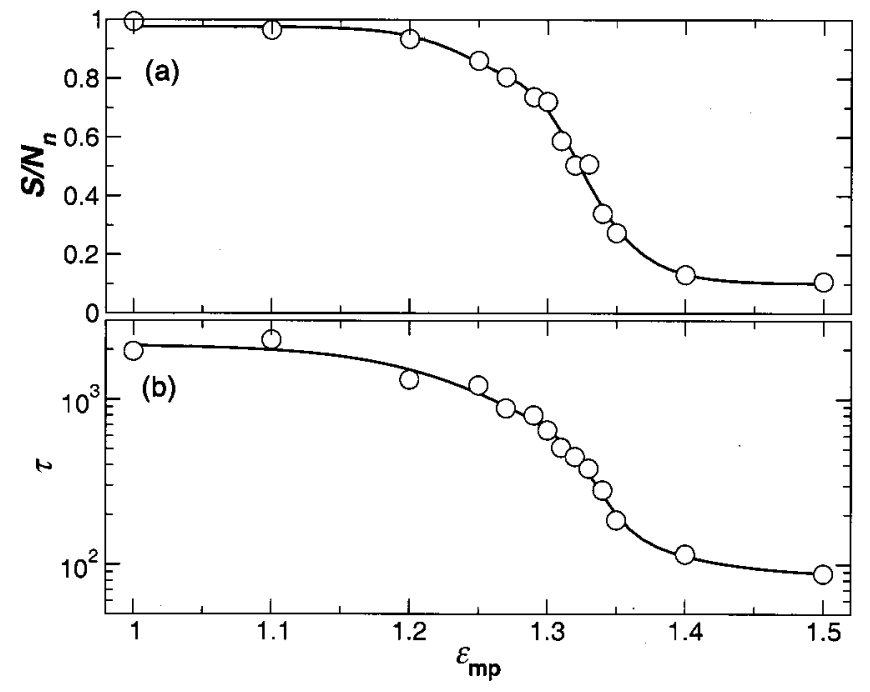

FIG. 7. (a) Cluster size $S / N_{n}$ normalized by the number of nanoparticles. (b) The characteristic lifetime $\tau_{R}$ of a bond between nanoparticles demonstrating the dynamic nature of the clusters.

weight average cluster size $S$. We define a cluster as the collection of neighboring nanoparticles, where a pair of nanoparticles are considered neighboring if their center-tocenter separation $\leqslant 3.96$, the location of the first minimum of the nanoparticle pair distribution function $g(r)$ in the maximally clustered states (i.e., $\varepsilon_{\mathrm{mp}}=1.0$ ), as shown in Fig. 4(b). We show $S / N_{n}$ in Fig. 7(a), where $N_{n}$ is the number of nanoparticles (distinct from $N_{p}$, the total number of force sites comprising the nanoparticles). We normalize by $N_{n}$ since $S / N_{n} \rightarrow 1$ when particles are in a single cluster, independent of the loading fraction. Figure 7(a) quantitatively shows that particles are relatively well-dispersed at large $\varepsilon_{\mathrm{mp}}$ with $S$ $\approx 3.5$ due to occasional contacts between the nanoparticles; this asymptotic value of $S$ must depend on $\phi$. At the opposite extreme, particles are almost entirely clustered in a single cluster at $\varepsilon_{\mathrm{mp}}=1$. The crossover between these states happens rapidly precisely in the range where $c_{V}^{\mathrm{pp}}$ has a maximum, justifying the identification of the maximum of $c_{V}^{\mathrm{pp}}$ as an approximate boundary between clustered and disperse states. While our results indicate $S / N_{n} \rightarrow 1$ when clustering occurs, there may be significant finite size effects. Given the relatively small number of nanoparticles we can simulate, it is possible that there is maximum characteristic cluster size that does not involve all of the nanoparticles.

In many systems,${ }^{33}$ clustering occurs through an intrinsically nonequilibrium process. In other words, once clustering occurs it is irreversible. Here the clustering is thermally reversible, and hence is a purely equilibrium phenomenon. In such a case, the clusters must be dynamic, and thus there is a characteristic lifetime of the "bond" between nanoparticles (where by "bond" we simply mean that nanoparticles neighbor each other according to the definition of the previous paragraph). We can quantify this by calculating the probability $P(t)$ that an initially bonded pair is bonded a time $t$ later. We then define the characteristic lifetime $\tau_{R}$ that a pair is neighboring by $P\left(t=\tau_{R}\right)=e^{-1}$, the relaxation time of $P(t)$. If the relaxation is exponential, $\tau_{R}$ corresponds to the expected form $P(t) \propto \exp \left(-t / \tau_{R}\right)$. However, when the clusters 


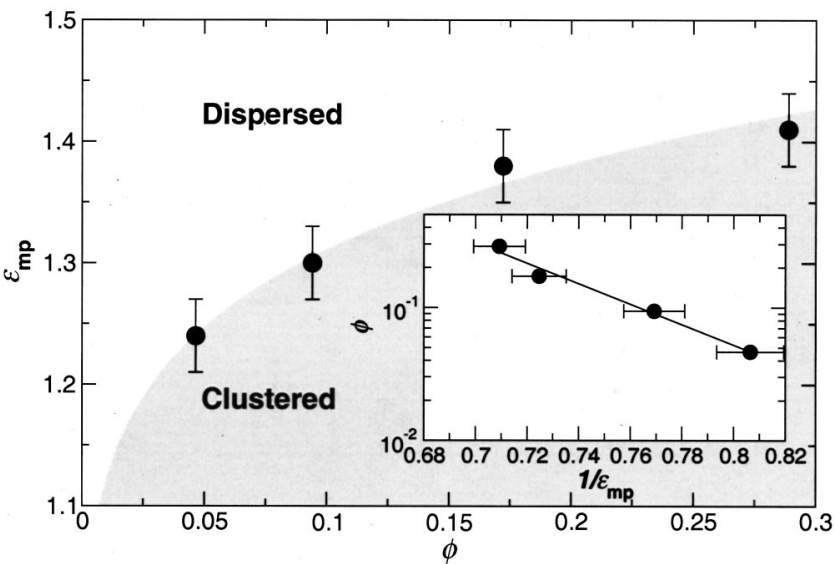

FIG. 8. (a) The "clustering diagram" of the nanoparticles, as a function of $\varepsilon_{\mathrm{mp}}$ and $\phi$. The boundary of the shaded region is determined by fitting the points using equation 5 , shown in the inset.

are longer lived, $P(t)$ (not shown) tends to be better described by a stretched exponential $P(t) \propto \exp \left(-t / \tau_{R}\right)^{\beta}$. Figure 7(b) shows $\tau_{R}$ increases dramatically when large clusters start to form, but $\tau_{R}$ remains finite even when nanoparticles are nearly completely clustered, demonstrating the equilibrium nature of the clustering. Similar equilibrium clustering has been documented for dipolar fluids in the absence of van der Waals attractions. ${ }^{34}$

We calculate the clustering behavior at fixed $T=2.0$ with variable $\varepsilon_{\mathrm{mp}}$ for additional values of $\phi$ by monitoring $u_{\mathrm{pp}}$ and $c_{V}^{\mathrm{pp}}$. For each $\phi$, we define the approximate boundary $\varepsilon_{\mathrm{mp}}^{*}$ between clustered and dispersed states by the value of $\varepsilon_{\mathrm{mp}}$ where $c_{V}^{\mathrm{pp}}$ is maximum. In this way, we obtain the clustering boundary in the $\left(\varepsilon_{\mathrm{mp}}, \phi\right)$ plane. Figure 8 shows the resulting "clustering diagram." We find that the boundary has an approximate exponential shape. The inset of Fig. 8 shows that

$$
\phi \sim \exp \left(-E_{0} / \varepsilon_{\mathrm{mp}}^{*}\right),
$$

with $E_{0}=17.7 . E_{0}$ provides a measure of the thermodynamic energy parameter controlling particle interactions. Note that $E_{0}$ should differ from $\varepsilon_{\mathrm{mp}}$, since $E_{0}$ is a thermodynamic measure that encompasses the cumulative effects caused by all of the specific interparticle interactions. This parameter characterizes the clustering transition, much like a $\chi$-parameter characterizes ordinary phase separation in polymer blends. The origin and relevance of this apparent Arrhenius behavior will become apparent in the following section when we discuss the $T$ dependence of the nanoparticle clustering.

\section{Effect of temperature}

The parameter most easily tuned in experimental or processing conditions is the temperature. Hence, it is of critical importance to understand the effect of $T$ on clustering. Generally speaking, increased $T$ favors states of higher entropy. The question then becomes the following: what configurations have a higher entropy? Dispersed nanoparticles are less ordered, and so we expect such configurations have a higher entropy, and thus will be favored as $T$ increases. However, if the nanoparticle loading is large, ordering of the nanopar-

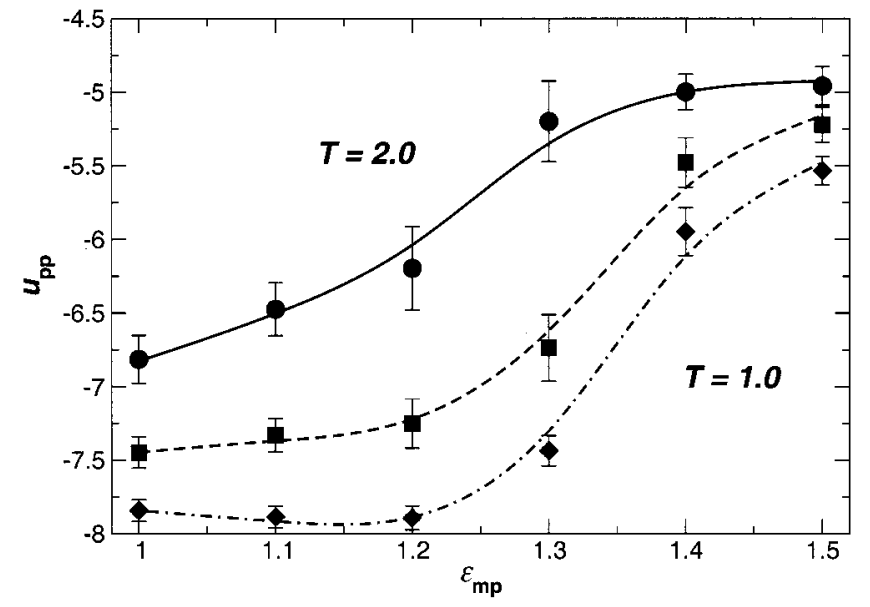

FIG. 9. Potential energy $u_{\mathrm{pp}}$ as a function of the coupling to the matrix, $\varepsilon_{\mathrm{mp}}$. The symbols are: $T=2.0(\boldsymbol{\bullet}), 1.5(\boldsymbol{\square})$, and $1.0(\bullet)$. The lines are a guide for the eye.

ticles might increase entropy, as is the case with the crystallization of colloidal systems. Additionally, there are a variety of complex systems for which increasing temperature can result in clustering, due to matrix properties. ${ }^{35}$

To build upon the observations of the previous section, Fig. 9 shows $u_{\mathrm{pp}}$ as a function of $\varepsilon_{\mathrm{mp}}$ along three different isothermal paths. The crossover between clustered and dispersed states occurs over a narrower range of $\varepsilon_{\mathrm{mp}}$ with decreasing $T$. Here again we estimate the boundary $\varepsilon_{\mathrm{mp}}^{*}$ in the $\left(T, \varepsilon_{\mathrm{mp}}\right)$ plane between clustered and dispersed states from the location of the maximum in $c_{V}^{\mathrm{pp}}$. Figure 10 clearly shows that increasing $\varepsilon_{\mathrm{mp}}$ or $T$ favors particle dispersion, suggesting that the parameters may serve largely complementary roles for determining the clustering state. This is expected, since $T$ is defined in terms of $\varepsilon$; given that there are 3 independent interaction parameters in the system, $T$ and $\varepsilon_{\mathrm{mp}}$ are not directly interchangeable. Below, we exploit the similarity in the behavior of $T$ and $\varepsilon_{\mathrm{mp}}$ to explain the exponential behavior shown in the inset of Fig. 8.

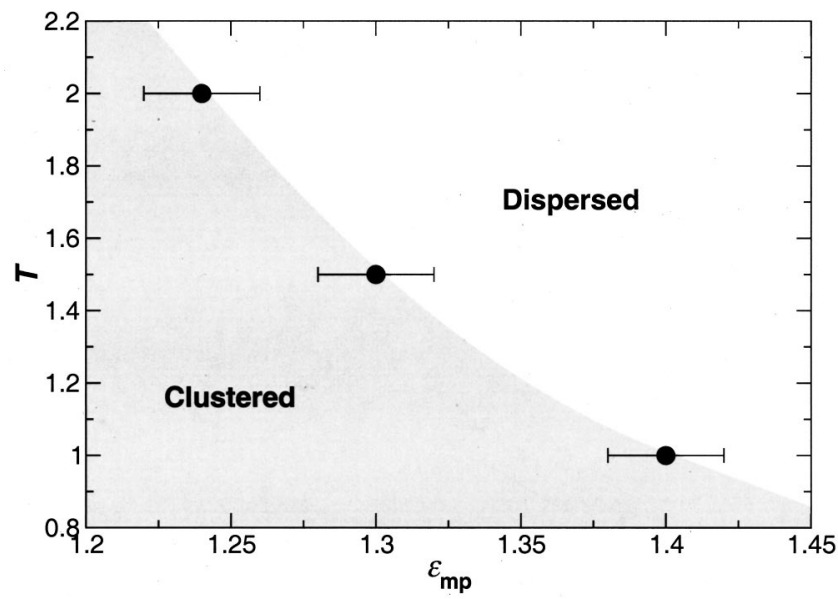

FIG. 10. The clustering diagram showing the $T$ and $\varepsilon_{\mathrm{mp}}$ dependence for a fixed loading fraction. Increasing either $T$ or $\varepsilon_{\mathrm{mp}}$ favors dispersion. The boundary is from a spline, and is only intended as a guide for the eye. 




FIG. 11. (a) Potential energy $u_{\mathrm{pp}}$ and (b) specific heat $c_{V}^{\mathrm{pp}}$ as a function of $T$ for loadings $\phi=0.046(\bigcirc), 0.094(\square), 0.172(\diamond)$, and $0.289(\triangle)$. Nanoparticles are clustered for low $u_{\mathrm{pp}}$, and the approximate boundary between clustered and dispersed states is given by the maximum in $c_{V}^{\mathrm{pp}}$. The lines are only a guide to the eye.

To further probe the effects of $T$ on the clustering properties, we simulate systems at variable $T$ for a fixed value of $\varepsilon_{\mathrm{mp}}=1.3$ at each $\phi$. This allows us to decouple the effects of $T$ from changes in the attraction between the nanoparticles and the polymers. Moreover, since models of particle clustering commonly focus on $(T, \phi)$ dependence, this offers an opportunity to explore the possible connections with these models. In Fig. 11 we show the behavior of $u_{\mathrm{pp}}$ and $c_{V}^{\mathrm{pp}}$ as a function of $T$ for several loadings. As expected, increasing $T$ favors dispersed states. However, the crossover between the states is not as clear from $u_{\mathrm{pp}}$ due to changes in the thermal vibrational energy. The crossover can be made clearer by subtracting the harmonic approximation to the vibrational energy given by $3 k_{\mathrm{B}} T / 2$. The crossover is most clearly shown by $c_{V}^{\mathrm{pp}}$, which again shows a maximum, facilitating the identification of the clustering boundary. A further examination of the behavior of the boundary is key to establishing the nature of the crossover between clustered and dispersed states.

We approximate the temperature $T^{*}$ of the boundary between clustered and dispersed states from the location of the maximum in $c_{V}^{\mathrm{pp}}$. Figure 12 shows the boundary is positively sloped, indicating that clustering occurs for large $\phi$ and low $T$. Comparing the behavior of the amplitude and location of the maximum of $c_{V}^{\mathrm{pp}}$ provides us with further evidence against phase separation via a first-order transition. From the shape of the boundary shown in Fig. 12, we see that, if the clustering mechanism were analogous to binary phase separation, the critical point must be at some $\phi>0.3$; at a critical $\phi$, the specific heat must diverge, although the divergence can be quite weak. ${ }^{36}$ However, we can see from Fig. 11(b) that the amplitude of the peak in $c_{V}^{\mathrm{pp}}$ decreases and becomes broader as we increase toward the possible critical value of $\phi$. For a binary system with a first-order phase transition, the amplitude of the peak in specific heat should increase as we approach the critical concentration, as observed in real mixtures $^{37}$ and pure systems. ${ }^{38}$

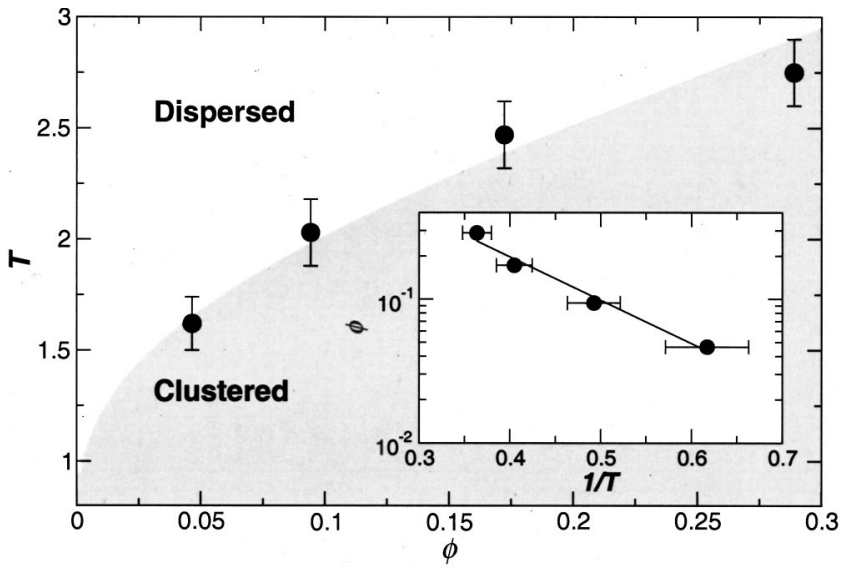

FIG. 12. The "clustering diagram" of the nanoparticles, as a function of $T$ and $\phi$. The boundary of the shaded region is determined by fitting the points using Eq. (6), shown in the inset. Note the similarity to the $(T, \phi)$ dependence shown in Fig. 8.

The decrease in the amplitude of the $c_{V}^{\mathrm{pp}}$ peak with increasing $\phi$ is consistent with the predicted behavior for an associating system. ${ }^{17}$ The model of equilibrium polymerization ${ }^{17}$ specifically predicts that the loci of specific heat maxima should shift location according to

$$
\phi \sim \exp \left(-E_{1} / T^{*}\right)
$$

The exponential temperature dependence derives from the Arrhenius temperature dependence of the rate constants describing the association and dissociation rate constants of the equilibrium particle association. We plot the clustering boundary in the inset of Fig. 12 to test for consistency with Eq. (6). Within the limits of uncertainty in our data, the clustering boundary can be described by Eq. (6), from which we obtain $E_{1}=6.9$. These findings suggest that the clustering transition in our system, and presumably in many similar real nanocomposite systems, is controlled by the same mechanism as simple associating systems. This observation provides a framework for rationalizing the behavior of many nanoparticle systems, which should in turn aid in the control of dispersion and nanocomposite properties.

As a final note, we point out that similarity to the predictions of dynamic clustering offers an explanation for the exponential behavior of the location of the maximum of $c_{V}^{\mathrm{pp}}$ observed as a function of $\varepsilon_{\mathrm{mp}}$, since $T$ and $\varepsilon_{\mathrm{mp}}$ play complementary roles. The difference is the values of $E_{0}$ and $E_{1}$ in Eqs. (5) and (6); specifically, $E_{0}>E_{1}$, indicating that the clustering transition is more sensitive to changes in the polymer-matrix interaction strength than $T$. Indeed, we know that $E_{0} \neq E_{1}$ from the fact that the boundary in Fig. 10 is not linear. The values of $E_{0}$ and $E_{1}$ may provide a useful point of departure to relate more mean-field approaches to the detailed model used here.

\section{NANOCOMPOSITE RHEOLOGY}

In the previous section, we gained some understanding of the mechanisms controlling particle dispersion in equilibrium. The behavior of nanocomposites under processing con- 


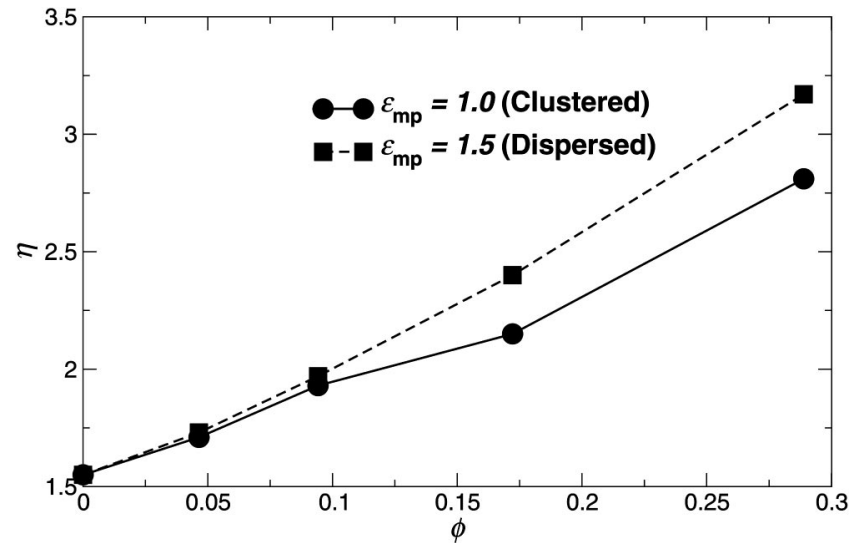

FIG. 13. Viscosity $\eta$ as a function of nanoparticle loading $\phi$ for a fixed shear rate $\dot{\gamma}=0.01$. Note that $\dot{\gamma}$ is small enough that initially clustered particles are not dispersed by the shear here.

ditions, where the system may experience strong shear, is also of critical importance. Hence, in this section, we focus on the effect of shear on nanocomposite clustering, as well as the effect of nanoparticle loading on the viscosity $\eta$.

One of the motivating factors behind the development of nanocomposite materials is the fact that substantial improvements in material properties can be obtained with very small amounts of added nanoparticles. Hence it is of great interest to examine how varying $\phi$ affects an important rheological property like viscosity $\eta$. We show $\eta$ as a function of $\phi$ for fixed $\dot{\gamma}=0.01$-small enough that the fluid is Newtonian (as we will soon show). Viscosity $\eta(\dot{\gamma})=\left\langle P_{x y}\right\rangle / \dot{\gamma}$, where $\left\langle P_{x y}\right\rangle$ is the average of the component of the pressure tensor along the flow and gradient directions of the shear. The error bars on $\eta$ indicate uncertainty due to fluctuations in time averaging; these fluctuations decrease with increasing $\dot{\gamma}$.

Figure 13 demonstrates that systems with well dispersed nanoparticles have larger viscosity. Previous studies ${ }^{9,11}$ have shown that $\eta$ is expected to increase as $\varepsilon_{\mathrm{mp}}$ increases, and thus it is not immediately clear whether the increase in viscosity shown in Fig. 13 is simply a result of the change of interaction strength, or if $\eta$ is also sensitive to the difference in particle clustering.

To address this question, we first calculate $\eta$ as a function of $\varepsilon_{\mathrm{mp}}$ for fixed $\phi=0.172$ - the loading fraction where we can first discern a difference in $\eta$ between clustered and dispersed states. Figure 14(a) shows that $\eta$ appears to approach nearly constant values at $\varepsilon_{\mathrm{mp}}=1$ and 1.5 , with a gradual crossover around $\varepsilon_{\mathrm{mp}} \approx 1.3$. In addition, we show the $c_{V}^{\mathrm{pp}}$ of the sheared systems in Fig. 14(b), indicating the crossover in clustering behavior occurs in the same range of $\varepsilon_{\mathrm{mp}}$ that $\eta$ changes between asymptotic regimes. Hence our results suggest that $\eta$ is in fact more sensitive to the state of particle clustering than to $\varepsilon_{\mathrm{mp}}$. Interactions must play a role in $\eta$, but this effect is less obvious here than in previous studies $^{9,11}$ since we vary $\varepsilon_{\mathrm{mp}}$ over a narrower range.

Why should particle dispersion result in increased viscosity? From hydrodynamic considerations, one expects the opposite effect- that a large, and extended rigid body embedded in a fluid results in a greater viscosity than a disperse collection of small rigid bodies. ${ }^{39}$ However, we previously

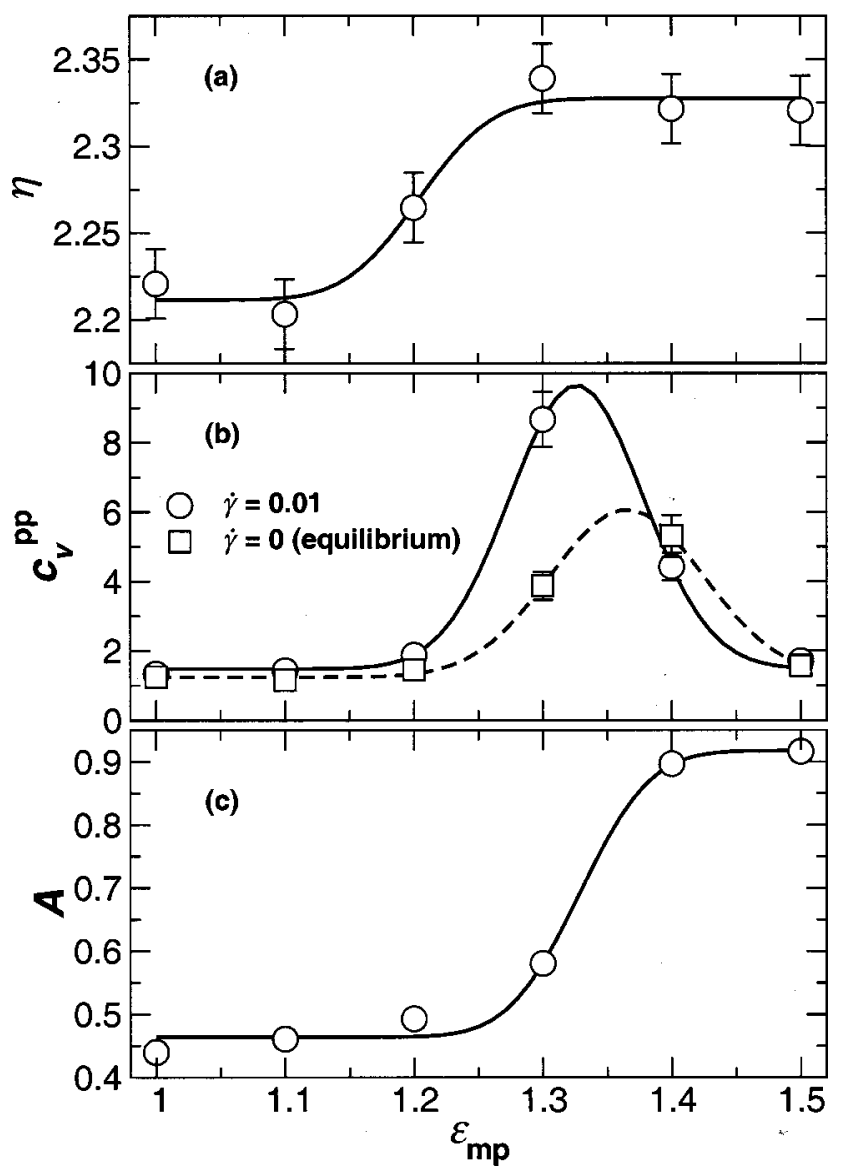

FIG. 14. (a) Viscosity $\eta$ as a function of $\varepsilon_{\mathrm{mp}}$ at fixed $T=2.0$ and $\phi$ $=0.172$. (b) Specific heat calculated according to Eq. (3) for the sheared configurations (circles, solid line) and equilibrium configurations (squares, broken line). Note the shift in the maximum, and its location compared with the crossover in the behavior of $\eta$. (c) The fraction of nanoparticle force sites in contact with a polymer, an estimate of the exposed surface area $A$. The lines serve only as guides to the eye.

showed that the clusters formed are dynamic in nature, and hence are not rigid. Moreover, changes in the polymer dynamics near the nanoparticle surface are known to play an important role in viscosity changes; previously we found that attractive surface interactions lead to a slowing of dynamics near the surface, which in turn results in an increased viscosity. ${ }^{9}$ For a fully dispersed configuration, the amount of exposed nanoparticle surface grows linearly with the number of nanoparticles; if clustering occurs, the amount of exposed surface grows sublinearly relative to the number of particles. Therefore, if the changes in $\eta$ are proportional to the amount of exposed nanoparticle surface, then $\eta$ should be larger for well-dispersed configurations than for clustered configurations. The correlation with the exposed surface area $A$-which we estimate by the fraction of nanoparticle force sites in contact with a chain-is demonstrated by Fig. 14(c). We define contact to mean that the separation between a monomer and a nanoparticle force site is $<1.5$, in accord with the first minimum of the pair distribution function. We point out that the large change in $A$ does not match exactly with the large change in $\eta$, suggesting that there remain less pronounced hydrodynamic effects that we cannot explain at the present time. Some progress in accounting for hydrody- 


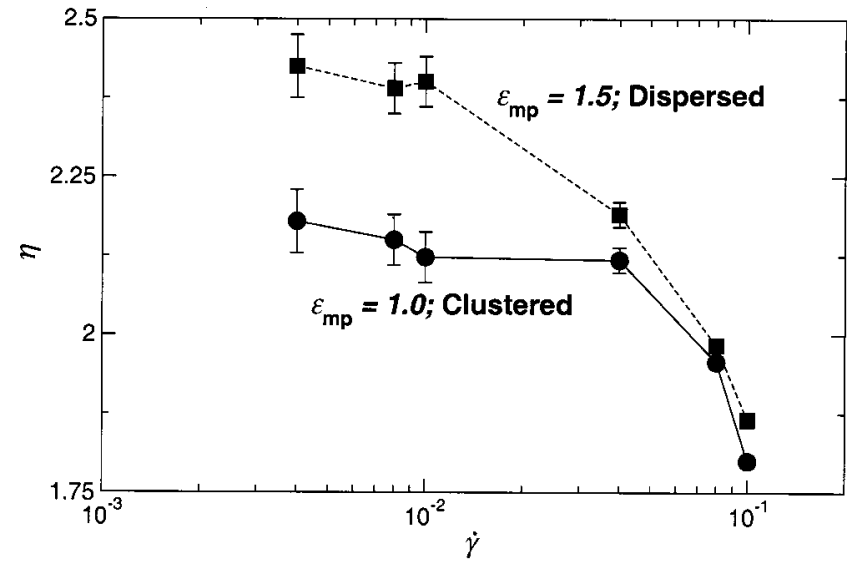

FIG. 15. Viscosity $\eta$ as a function of shear rate $\dot{\gamma}$ for $\phi=0.172$.

namic effects on viscosity in a system with a single nanoparticle has been made, ${ }^{11}$ but these results do not account for changes due to clustering in multinanoparticle systems.

We also point out that Fig. 13 shows that the differences in viscosity are extremely small for $\phi \leqq 0.15$-well above loadings that are expected to be relevant for nanocomposite applications. There are variety of possible sources for the large loading that is needed to obtain significant differences in $\eta$, such as the nanoparticle geometry and the complexity of the interactions. Given the expected importance of surface area, simulations of claylike plate nanoparticles using the same interactions as used for the nanoparticles have been conducted; the surface-to-volume ratio of a single platelike nanofillers is far greater than for a nearly spherical nanoparticle. Preliminary results from these simulations show that differences in $\eta$ comparable to the largest $\phi$ studied here can be obtained with only $\phi \approx 5 \% .{ }^{40}$ This supports the importance of nanoparticle surface area, and hence the properties of the interfacial polymers, on the ultimate bulk properties. The aspect ratio may also play a key role, since the ability of the sheet to diffuse through the melt is dramatically different in the directions perpendicular and parallel to the sheet normal. These are areas for future investigation.

Our results for $c_{V}^{\mathrm{pp}}$ indicate a shift in the clustering diagram under shear. Figure 14(b) shows $c_{V}^{\mathrm{pp}}$ for both the sheared and equilibrium systems. While the appropriate definition of $c_{V}^{\mathrm{pp}}$ in a nonequilibrium system is not clear, we present it simply as an indication of the fluctuations in nanoparticle potential energy, and hence the fluctuations in nanoparticle contacts. By comparing the results for the systems with and without shear, it is clear that shearing expands the region where particles tend to disperse. Indeed, for clustered configurations near the crossover point, sufficient shear will disperse the particles. Hence, shear favors particle dispersion in this system.

Finally, we consider $\eta$ as a function of the shear rate $\dot{\gamma}$ for the same equilibrium state points $(\phi=0.172, T=2.0)$ with monomer-particle interaction strengths $\varepsilon_{\mathrm{mp}}=1.0$ and 1.5. This allows us to test for differences in the response to shear that result from the state of equilibrium clustering. Figure 15 shows $\eta$ as a function of $\dot{\gamma}$. The composite approaches Newtonian behavior (i.e., $\eta$ independent of $\dot{\gamma}$ ) for small $\dot{\gamma}$, while for $\dot{\gamma} \geq 10^{-2}$, shear thinning occurs. Such thinning is expected since a pure melt of 10 -mer chains is known to undergo shear thinning in this range, ${ }^{30}$ largely due to alignment of these short, unentangled chains. The viscosity of the dispersed configuration is larger than that of the clustered configuration over the range of $\dot{\gamma}$ studied, although at larger $\dot{\gamma}$ the difference is small. The viscosity difference is expected since we are far from any phase boundary, and hence the dispersions (or clusters) are highly stable. However, since we have observed shear induced dispersion for systems near the clustering transition line, we thus expect that sufficiently large shear will disperse even the highly stable equilibrium clusters.

\section{CONCLUSION}

We have investigated the mechanism of nanoparticle clustering in a simple model polymer nanocomposite, as well as the interplay between clustering and the shear viscosity of the system. The crossover between clustered and dispersed states is consistent with the predictions made for particle association by a thermoreversible process, and hence does not seem to match with expectations of phase separation in a binary mixture, over the temperature range considered. While this suggests that the nanoparticle clustering is akin to equilibrium polymerization, we also point out that, without appropriate finite size studies, our results cannot rule out the possibility that the nanoparticles are crystallizing, as occurs in micelle systems above a certain volume fraction ${ }^{8}$ and protein solutions. ${ }^{41}$ While further study is still needed, we believe that the patchiness of the potential plays an important role in these results. It has been argued that such patchiness is crucial in the self-assembly of globular proteins into higher-order structures; ${ }^{42}$ these proteins have a size similar to nanoparticles, and exhibit relatively short range interactions relative their size, like the nanoparticles simulated here. Aside from thermodynamic considerations, the dynamics of the nanoparticle clustering and break-up remains an open question, although some progress has been made in this direction for clay systems. ${ }^{43}$ From the standpoint of molecular simulation, this is a particularly challenging aspect, given the very slow dynamics of the nanoparticle clustering. However, by exploiting the similarity of clustering with simpler associating systems, a better approach may be to focus on the clustering dynamics of simple systems, where longer time scales are more accessible.

Symmetric nanoparticles are one of the simpler examples of nanoscale additives to polymeric materials. Given the burgeoning activity with exfoliated clay sheets and carbon nanotubes, it is critical that future studies focus on how geometry affects the clustering properties in these systems. The factors that control sheet stacking or fiber bundling may be markedly different from what we have observed. As mentioned in the previous section, it appears that the surface-tovolume and the aspect ratios play as crucial a role as dispersion in the rheological properties. Finally, future studies should also consider the effects of ionic and bonding interactions that could lead to significant changes in the mechanisms of particle clustering. 


\section{ACKNOWLEDGMENTS}

We wish to thank E. Amis, K. Anderson, B. Farmer, C. Han, E. Hobbie, T. Schrøder, G. Smith, R. Vaia, and K. Van Workum for useful discussions.

${ }^{1}$ G. Wypych, Handbook of Fillers (ChemTec, Toronto, 1999).

${ }^{2}$ M. C. Roco, S. Williams, and P. Alivisatos, in, Nanotechnology Research Directions: IWGN Workshop Report Vision for Nanotechnology in the Next Decade (Kluwer Academic, Dordrecht, 2000).

${ }^{3}$ R. A. Vaia and E. P. Giannelis, MRS Bull. 26, 394 (2001).

${ }^{4}$ J. N. Israelachvili, Intermolecular and Surface Forces (Academic, New York, 1992).

${ }^{5}$ O. W. Webster, Science 25, 887 (1991)

${ }^{6}$ Q. Ying, J. Maracek, and B. Chu, J. Chem. Phys. 101, 2665 (1994); T. Rudalevige, A. H. Francis, and R. Zand, J. Phys. Chem. A 102, 9797 (1998); S. Nath, H. Pal, D. K. Palit, A. V. Sapre, and J. P. Mittal, J. Phys. Chem. B 102, 10158102 (1998).

${ }^{7}$ M. Djikstra, J. P. Hansen, and P. A. Madden, Phys. Rev. Lett. 75, 2236 (1995); T. A. Witten, J. Phys. (Paris) 49, 1055 (1988); C. E. Iona, T. Aberle, and W. Burchard, Macromolecules 34, 326 (2001).

${ }^{8}$ R. G. Larson, Chem. Eng. Sci. 49, 2833 (1994).

${ }^{9}$ F. W. Starr, T. B. Schrøder, and S. C. Glotzer, Phys. Rev. E 64, 021802 (2001); Macromolecules 35, 4481 (2002).

${ }^{10}$ M. Vacatello, Macromolecules 34, 1946 (2001).

${ }^{11}$ G. D. Smith, D. Bedrov, L. Li, and O. Byutner, J. Chem. Phys. 117, 9478 (2002).

${ }^{12}$ D. Brown, P. Mélé, S. Marceau, and N. D. Albérola, Macromolecules 36, 1395 (2003).

${ }^{13}$ D. Gersappe, Phys. Rev. Lett. 89, 058301 (2002).

${ }^{14}$ V. V. Ginzburg, C. Singh, and A. C. Balazs, Macromolecules 33, 1089 (2000).

${ }^{15}$ M. H. J. Hagen, E. J. Meijer, G. C. A. M. Mooij, D. Frenkel, and H. N. W. Lekkerkerker, Nature (London) 365, 425 (1993).

${ }^{16}$ V. J. Anderson and H. N. W. Lekkerkerker, Nature (London) 416, 811 (2002); H. N. W. Lekkerkerker, Physica A 244, 227 (1997).

${ }^{17}$ J. Dudowicz, K. F. Freed, and J. F. Douglas, J. Chem. Phys. 111, 7116 (1999); 112, 1002 (2000).

${ }^{18}$ R. B. Bird, C. F. Curtiss, R. C. Armstrong, and O. Hassager, Dynamics of Polymeric Liquids: Kinetic Theory, Vol. 2 (Wiley, New York, 1987).

${ }^{19}$ G. S. Grest and K. Kremer, Phys. Rev. A 33, 3628 (1986).

${ }^{20}$ J. W. Rudisill and P. T. Cummings, Rheol. Acta 30, 33 (1991).

${ }^{21}$ J.-C. Donnet, R. C. Bansal, and M.-J. Wang, Eds., Carbon Black: Science and Technology (Marcel Dekker, New York, 1993).

${ }^{22}$ G. Carrot, J. C. Valmalette, C. J. G. Plummer, S. M. Scholz, J. Dutta, H. Hofmann, and J. G. Hilborn, Colloid Polym. Sci. 276, 853 (1998); D. H. Cole, K. R. Shull, P. Baldo, and L. Rehn, Macromolecules 32, 771 (1999); F. Carusoa and H. Mohwald, Langmuir 15, 8276 (1999); K. V. Sarathy, K. S. Narayan, J. Kim, and J. O. White, Chem. Phys. Lett. 318, 543 (2000);
D. I. Gittins and F. Caruso, Adv. Mater. (Weinheim, Ger.) 12, 1947 (2000); K. J. Watson, J. Zhu, S. T. Nguyen, and C. A. Mirkin, Pure Appl. Chem. 72, 67 (2000).

${ }^{23}$ K. A. Barnes, A. Karim, J. F. Douglas, A. I. Nakatani, H. Gruell, and E. J Amis, Macromolecules 33, 4177 (2000); B. McCarthy, J. N. Coleman, S. A. Curran, A. B. Dalton, A. P. Davey, Z. Konya, A. Fonseca, J. B. Nagy, and W. J. Blau, J. Mater. Sci. Lett. 19, 2239 (2000); Y. Okumura, K. Ito, R. Hayakawa, and T. Nishi, Langmuir 16, 10278 (2000); V. Lordi and N. Yao, J. Mater. Res. 15, 2770 (2000); R. M. Ajayan, L. S. Schadler, C. Giannaris, and A. Rubio, Adv. Mater. (Weinheim, Ger.) 12, 750 (2000); D. Qian, E. C. Dickey, R. Andrews, and T. Rantell, Appl. Phys. Lett. 76, 2868 (2000).

${ }^{24}$ A. Karim, D. W. Liu, J. F. Douglas, A. I. Nakatani, and E. J. Amis, Polymer 41, 8455 (2000); A. I. Nakatani, W. Chen, R. G. Schmidt, G. V. Gordon, and C. C. Han, ibid. 32, 3713 (2001); C. Roberts, T. Cosgrove, R. G. Schmidt, and G. V. Gordon, Macromolecules 34, 538 (2001).

${ }^{25}$ F. J. Feher, D. Soulivong, A. G. Eklund, and K. D. Wyndham, Chem. Commun. (Cambridge) 13, 1185 (1997); J. J. Schwab and J. D. Lichtenhan, Appl. Organomet. Chem. 12, 707 (1998).

${ }^{26}$ M. Tuckerman, B. J. Berne, and G. J. Martyna, J. Chem. Phys. 97, 1990 (1992).

${ }^{27}$ M. P. Allen and D. J. Tildesley, Computer Simulation of Liquids (Oxford University Press, Oxford, 1987).

${ }^{28}$ D. Di Tolla and M. Ronchetti, Phys. Rev. E 48, 1726 (1993).

${ }^{29}$ S. T. Cui, P. T. Cummings, and H. D. Cochran, J. Chem. Phys. 104, 255 (1996)

${ }^{30}$ Z. Xu, J. J. de Pablo, and S. Kim, J. Chem. Phys. 102, 5836 (1995).

${ }^{31}$ H. Jónsson and H. C. Andersen, Phys. Rev. Lett. 60, 2295 (1988); F. Sciortino, A. Geiger, and H. E. Stanley, ibid. 96, 3857 (1992); C. Donati, S. C Glotzer, P. H. Poole, W. Kob, and S. J. Plimpton, Phys. Rev. E 60, 3107 (1999).

${ }^{32}$ K. Binder, Rep. Prog. Phys. 50, 783 (1987).

${ }^{33}$ P. Meakin, Phase Transitions 12, 336 (1988).

${ }^{34}$ P. I. C. Teixeira, J. M. Tavares, and M. M. Telo da Gama, J. Phys.: Condens. Matter 12, R411 (2000).

${ }^{35}$ S. C. Greer, Annu. Rev. Phys. Chem. 53, 173 (2002).

${ }^{36}$ H. E. Stanley, Introduction to Phase Transitions and Critical Phenomena (Oxford University Press, Oxford, 1971).

${ }^{37}$ D. Jacobs (unpublished); Kh. S. Abdulkadirova, A. Kostrowicka Wyczalkowska, M. A. Anisimov, and J. V. Sengers, J. Chem. Phys. 116, 4597 (2002).

${ }^{38}$ A. Kostrowicka Wyczalkowska, Kh. S. Abdulkadirova, M. A. Anisimov, and J. V. Sengers, J. Chem. Phys. 113, 4985 (2000).

${ }^{39}$ J. Bicerano, J. F. Douglas, and D. A. Brune, Rev. Macromol. Chem. Phys. C39, 561 (1999).

${ }^{40}$ F. W. Starr, R. A. Vaia, and B. L. Farmer (unpublished).

${ }^{41}$ C. R. Berland et al., Proc. Natl. Acad. Sci. U.S.A. 89, 1214 (1992).

${ }^{42}$ A. Lomakin et al., Proc. Natl. Acad. Sci. U.S.A. 96, 9465 (1999).

${ }^{43}$ A. Sinsawat, K. Anderson, R. A. Vaia, and B. L. Farmer (preprint). 